\title{
Investigations on noise sources on a contra-rotating axial fan with different modifications
}

\author{
Ralph Krause ${ }^{1, *}$, Christian Friebe ${ }^{1}$, Michael Kerscher $^{2}$, Christof Puhle $^{3}$ \\ ${ }^{1}$ INSTITUT FÜR LUFT- UND KÄLTETECHNIK gGmbH, Bertolt-Brecht-Allee 20, 01309 Dresden, Germany \\ ${ }^{2}$ GFAI TECH GmbH, Volmerstraße 3, 12489 Berlin, Germany \\ ${ }^{3}$ GFAI Gesellschaft zur Förderung angewandter Informatik e.V., Volmerstraße 3, 12489 Berlin, Germany
}

\begin{abstract}
An Acoustic Camera was applied to examine modifications of fan blade designs regarding their noise emissions. A so-called rotational beamforming algorithm allows for the detection of sound sources on the rotating blades by using a virtual rotation of the microphones. Depending upon the frequency different sources could be localized. Both the leading and the trailing edge were modified. This paper shows the performed modifications and tests with the Acoustic Camera. It also presents first results and gives an outlook on future work.
\end{abstract}

\section{Introduction}

Axial blowing fans are commonly known in many fields of applications. By using axial fans, swirls occur at the trailing edge of the blades due to the working principle. These swirls are unwanted in most cases and may have unfavourable influence on subsequent devices, e.g. higher pressure drop or lower heat transfer coefficient. As the static pressure rise is an evaluation criterion for the fan efficiency, there are different possibilities for converting the dynamic pressure of the swirl into a static pressure rise. The most common application for rising the efficiency is the installation of outlet guide vanes.

The installation of contra-rotating axial fans (CRF) is another well-known opportunity to increase the efficiency of a fan. Beside the high power density and the high efficiency it should be noted that these fans are known for problematic noise behaviour. This is induced by the interaction of both wheels with different directions of rotation.

The aim of the project is to investigate the influence of different suggestions of literature on how to reduce the noise emission of fans based on modifications of the blade design.

An Acoustic Camera was applied to examine various modifications of fan blade designs regarding their noise emissions. A so-called rotational beamforming algorithm allows the detection of noise sources on the rotating blades by using a virtual rotation of the microphones. Depending upon the frequency, different sources could be localised [1].

Both the leading and the trailing edge were modified. The leading edge was shaped with a sinusoidal structure and is supposed to lead to a lower leading edge noise, which is mostly induced by impinging vortices [2-7]. The trailing edge was modified using serrations. These serrations are supposed to lead to vortices at higher frequencies [8-10].

This paper shows the performed modifications and tests with the Acoustic Camera. It also presents first results and gives an outlook on future work and usage.

\section{Working Principle Contra-Rotating Fan}

Due to the Euler equation the specific work is defined by

$$
Y=u^{\prime \prime} c_{u}^{\prime \prime}-u^{\prime} c_{u}^{\prime}
$$

For an axial fan stage obviously $u^{\prime \prime}=u^{\prime}=u$, which transforms eq. (1) to

$$
Y=u\left(c_{u}^{\prime \prime}-c_{u}^{\prime}\right),
$$

with $u$ being the amount of the circumferential speed, $c_{u}$ the component of the fluid velocity vector $\boldsymbol{c}$ with the same direction of $\boldsymbol{u}$ and ' as inlet and " as outlet symbols.

According to eq. (2) a pressure rise in an axial impeller stage causes a change in the outflow $c_{A, u}^{\prime \prime} \neq 0$ compared to the inlet conditions $c_{A, u}^{\prime}=0$. The second impeller, with an opposite direction of rotation, shall be designed without outlet swirl as there is already a swirl at the inlet $c_{B, u}^{\prime}<0$. Fig 1 represents the velocity components for a contra rotating axial fan stage without outlet swirl, defined by $c_{u}^{\prime \prime}=0$.

\footnotetext{
*orresponding author: ralph.krause@ilikdresden.de
} 


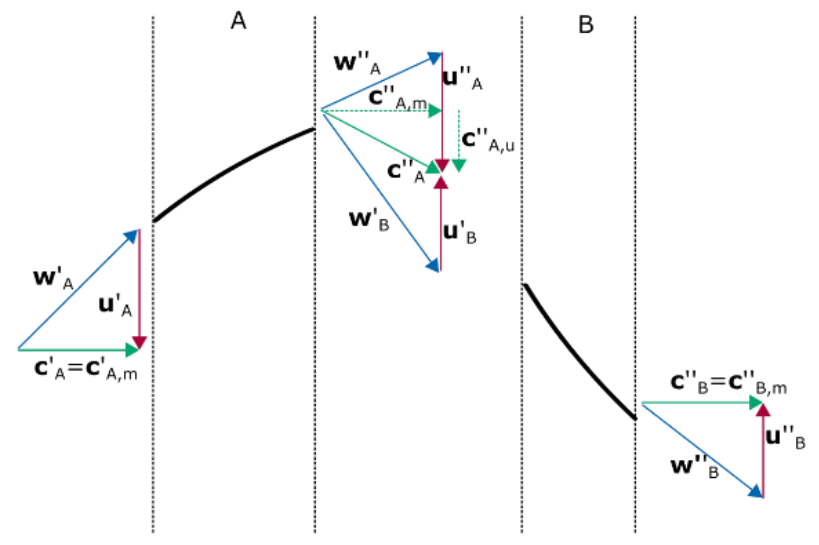

Fig 1. Schematic representation of the velocity components

The fan configuration used in this paper corresponds to the schematic drawing as presented in Fig 2. Each impeller (A, B) is driven with a separate motor. Stationary components and rotating components are differently coloured. Stationary components are presented in black and rotating components are coloured in blue and red, according to their direction of rotation.

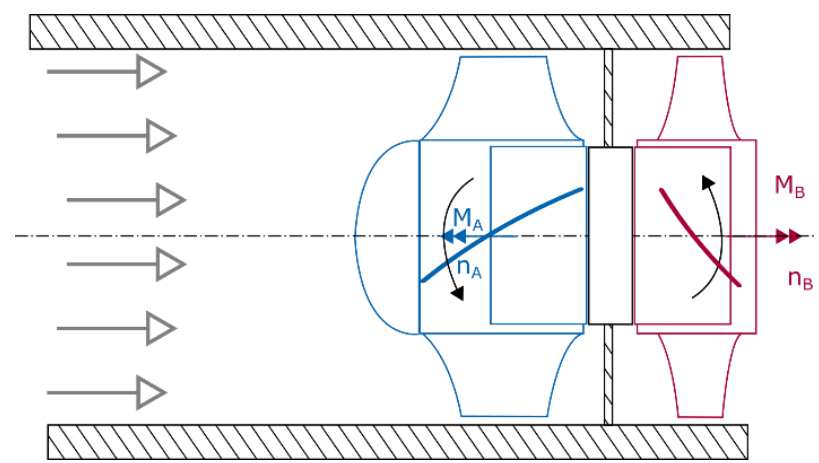

Fig 2. Schematic representation of the CRF-construction with one drive for each impeller

\section{Rotational beamforming}

\subsection{Functional Beamforming}

Let $M$ be the number of microphones of a phased array and $C \in C^{M}$ the corresponding matrix of auto- and crossspectra resulting from an averaged short-time FFT of a measurement. Then, in the frequency domain formulation of delay-and-sum beamforming (FDBF), the map value at a point $x_{i} \in R^{3}$ is given by

$$
B\left(x_{i}\right)=h^{H}\left(x_{i}\right) \operatorname{Ch}\left(x_{i}\right)
$$

where the superscript $H$ denotes the conjugate transpose and $h^{H}\left(x_{i}\right) \in C^{M}$ is the steering vector of type I of $x_{i}$ [11]. The auto-spectra in the diagonal of $C$ do not contain any information about phase differences between the signals. Consequently, they are often removed in order to reduce the error introduced by uncorrelated background noise.

Caused by fundamental properties of the beamforming approach and the chosen array geometry, the map $B$ is a convolution of the actual source distribution with a point spread function leading to high side-lobe levels and limiting the dynamic range of the acoustic map.

To overcome this problem deconvolution methods have been proposed to decompose $\mathrm{C}$ into parts representing individual sources [12-14]. Among them, functional beamforming (FB) is particularly useful to increase the dynamic range. In addition, it allows for a combination with the decomposition approach of orthogonal beamforming (OB).

Since $C$ is self-adjoint by definition, there exist $\lambda_{1}, \ldots, \lambda_{M} \in R$ and an orthogonal basis $u_{1}, \ldots, u_{M} \in C^{M}$ such that

$$
C=\sum_{m=1}^{M} \lambda_{m} u_{m} u_{m}^{H}
$$

Each summand $\lambda_{m} u_{m} u_{m}^{H} \in C^{M \times M}$ is called component of $C$. Suppose that $C$ is positive definite. Then $\lambda_{1}, \ldots, \lambda_{M}>0$ and

$$
C^{\frac{1}{v}}:=\sum_{m=1}^{M} \lambda_{m}^{\frac{1}{v}} u_{m} u_{m}^{H}
$$

is well defined for $v \in N, v \geq 1$. Now the value of the functional beamforming map of order $v$ at $x_{i}$ can be defined as

$$
B_{\mu}^{v}\left(x_{i}\right):=\left(h^{H}\left(x_{i}\right) C^{\frac{1}{v}} h\left(x_{i}\right)\right)^{v}
$$

In many cases throughout this article we assume $\lambda_{1} \geq \cdots \geq \lambda_{M}$ and consider only the first $\mu \in N, \mu \geq 1$ components, i.e. we use the reduced functional beamforming map $B_{\mu}^{v}$ of order $v$ :

$$
\begin{aligned}
B_{\mu}^{v}\left(x_{i}\right) & :=\left(h^{H}\left(x_{i}\right) C^{\frac{1}{v}} h\left(x_{i}\right)\right)^{v}, C^{\frac{1}{v}} \\
& :=\sum_{m=1}^{M} \lambda_{m}^{\frac{1}{v}} u_{m} u_{m}^{H}
\end{aligned}
$$

\subsection{Virtual Array Rotation}

The methods of the previous section are based on the assumption that the phased array and the measured object are fixed spatially to each other. Since this is obviously not the case when measuring a rotating fan, we determine the signals of a virtual microphone array that is fixed in the coordinate system of the fan. For each point in time $t$ let $p_{1}^{v}(t), \ldots p_{M}^{v}(t) \in R^{3}$ denote the microphone positions of the virtual array in the coordinate system of the array measurement at $p_{1}(t), \ldots p_{M}(t) \in R^{3}$. The signal value $s_{i}^{v}\left(t_{0}\right)$ of the $i$-th virtual microphone at $t_{0}$ is determined as follows. We compute the two nearest neighbours $p k$, $p l$ of $p_{i}^{v}$ among the measurement positions and set

$$
s_{1}^{v}\left(t_{0}\right)=\frac{\left\|p_{k}-p_{1}^{v}\left(t_{0}\right)\right\|_{2} s_{l}\left(t_{0}\right)+\left\|p_{l}-p_{1}^{v}\left(t_{0}\right)\right\|_{2} s_{k}\left(t_{0}\right)}{\left\|p_{k}-p_{1}^{v}\left(t_{0}\right)\right\|_{2}+\left\|p_{l}-p_{1}^{v}\left(t_{0}\right)\right\|_{2}}
$$

where $s_{k}\left(t_{0}\right)$ denotes the signal value of the $j$-th measurement microphone at $t_{0}$. 


\section{Measurement Setup}

\subsection{Test Samples}

The fan under investigation has a tip diameter of $300 \mathrm{~mm}$ and rotational speeds of $32 \mathrm{~s}^{-1}\left(1920 \mathrm{~min}^{-1}\right)$ and $20 \mathrm{~s}^{-1}$ $\left(1200 \mathrm{~min}^{-1}\right)$ for the first and the second impeller, respectively. It is designed for an air-flow rate of $0.486 \mathrm{~m}^{3} \mathrm{~s}^{-1}\left(1750 \mathrm{~m}^{3} \mathrm{~h}^{-1}\right)$ and for a total pressure differences of $180 \mathrm{~Pa}(120 \mathrm{~Pa}$ at the first and $60 \mathrm{~Pa}$ at the second impeller).

The design of the fan has been carried out using the program CFturbo [15]. The design in CFturbo follows a strict conceptual approach that considers 1D-balance equations, empirical correlations and airfoil theory. The geometry description is completely parametric and allows therefore adjustments of any detail. Each impeller's boundary conditions are set according to the power distribution $(120 \mathrm{~Pa}$ and $60 \mathrm{~Pa})$ and the respective speed in the conceptual design process. The same applies to the pre-swirl that is zero for the first impeller. For the second impeller the pre-swirl is equal to the swirl produced by the first impeller.

The result of the design are blades with straight leading and trailing edges for both impellers although sweeping of the blades would have been possible, too. This modification is not applied since some means of trailing and leading edge treatment shall be executed afterwards. These modifications shall be done on straight edges to compare the influence more easily.

The blades haven been modified due to acoustical reasons. Those of impeller A have a modified trailing edge with serrations according to Carolus [8], Catalano [9] and Howe [10]. Both, the unmodified standard blades and the blades with modified trailing edge are presented in Fig. 3.
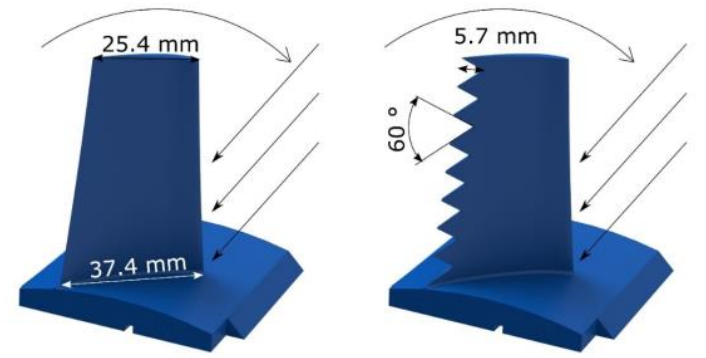

Fig. 3. Blades of impeller A in standard configuration (left) and with modified trailing edge (right)

Another attempt is the modification of the leading edge. Different types of those edges are discussed by Corsini and Delibra [2], Sodermann [6], Hansen et al [3], Hersh et al. [4], Polacsek et al. [5] and Johari et al. [7]. The design study $4 \mathrm{~L}$ of Johari et al. [7] with a sinusoidal leading edge is used within this study. Both types of blades for impeller B are depicted in Fig. 4.

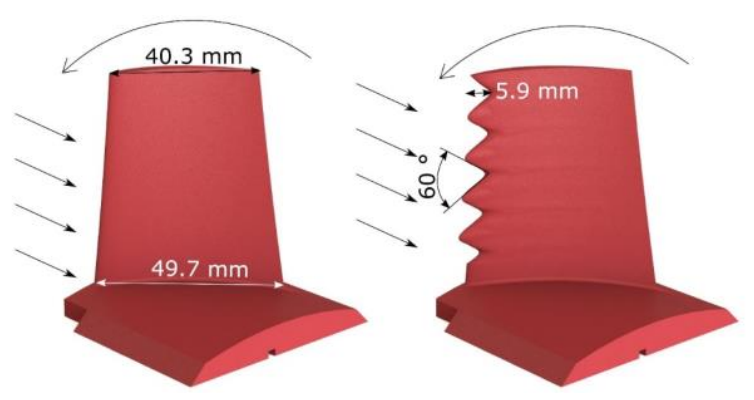

Fig. 4. Blades of impeller B in standard configuration (left) and with modified leading edge (right)

These four different types of blades have been permutated to four different samples. Sample 1 has unmodified blades at both impellers. Sample 2 consists of modified impeller A and unmodified impeller B and vice versa in Sample 3. Both impellers in Sample 4 have modified blades (see Fig. 5).

Both impellers are mounted at stand-alone drives. These drives have opposite directions of rotation and are controlled separately. Each sample has seven blades at impeller A and five blades at impeller B.
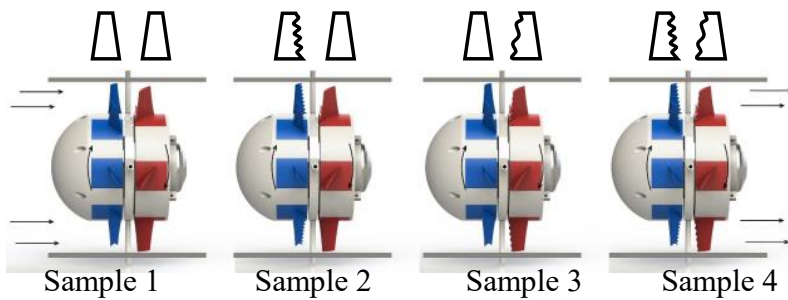

Fig. 5. Design studies under examination, from left to right: Sample 1 with straight edges for impeller A and B, Sample 2 with serrations at the trailing edge of impeller A, Sample 3 with sinusoidal leading edge of impeller B, Sample 4 serrations at trailing edge of impeller $A$ and sinusoidal leading edge of impeller B.

\subsection{Test Stand}

The measurements are carried out as described in [16] at a test stand complying with ISO 5081 [17]. Category C was selected as mode of installation, implying that there is a pipe at the suction side and a free outlet at the pressure side of the fan. Deviating to the standard, a silencer was installed in front of the fan. The installation of the silencer is necessary for the acoustic measurements but shall have no influence on the fan operation itself. A schematic drawing of the test stand is presented in Fig. 6.

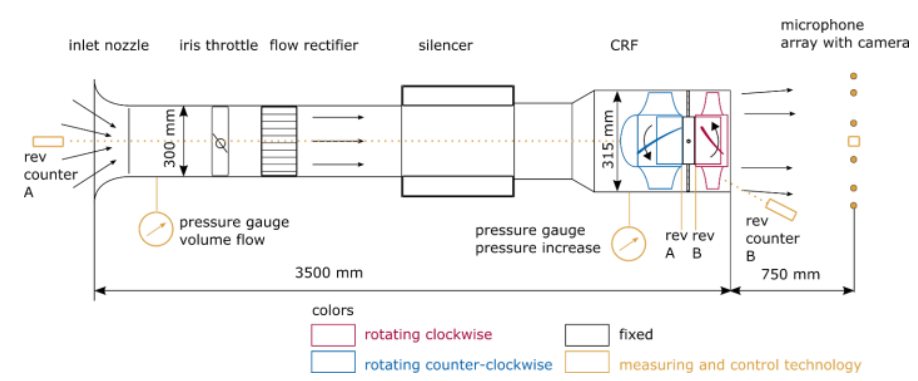

Fig. 6. schematic drawing of the test stand 
To meet the design point of fan operation the pressure drop is adjustable by means of a throttle valve. The flow rate was measured by means of the inlet nozzle and the static pressure rise utilizing the static pressure difference at the suction side of the fan against the environment. The rectifier was installed to achieve a constant and equal velocity profile. The silencer is employed for reducing disturbances by reflection of noise towards the suction side of the fan. The fan was hold by a 4-arm hub.

Fig. 7 shows the measurement setup in a semianechoic room. An Acoustic Camera was placed at a distance of $0.75 \mathrm{~m}$ looking directly towards the pressure side of the fan. In order to receive precise results an accurate positioning of the array is needed. A concentric relationship between the microphone ring and the air duct as well as parallel relationship between the plane of the fan outlet and the plane of the microphones had been established by measuring and adjusting the distances between the hub center and the microphones.

As the rotational beamforming algorithm uses a virtual microphone rotation, symmetric array geometries shall be preferred. A 48-channel ring-array with a diameter of 0.75 $\mathrm{m}$ was used here. The rotation speeds of the impellers were recorded with two laser rpm-meter facing towards the impellers from either side of the test stand.

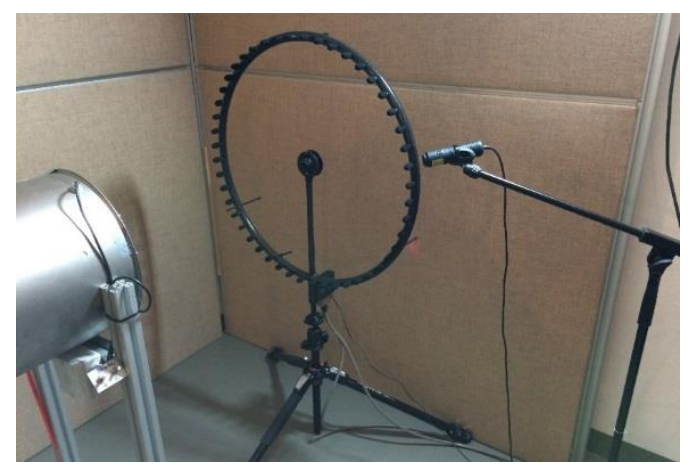

Fig. 7. Microphone array in front of the pressure side of the $\mathrm{CRF}$

\section{Measurement results}

\subsection{Performance}

The results obtained by the performance tests for Sample 1 and Sample 2 are presented in Fig. 8. Throughout the measurement the rotational speeds have been fixed at $n_{A}=32 s^{-1}$ and $n_{B}=20 s^{-1}$.

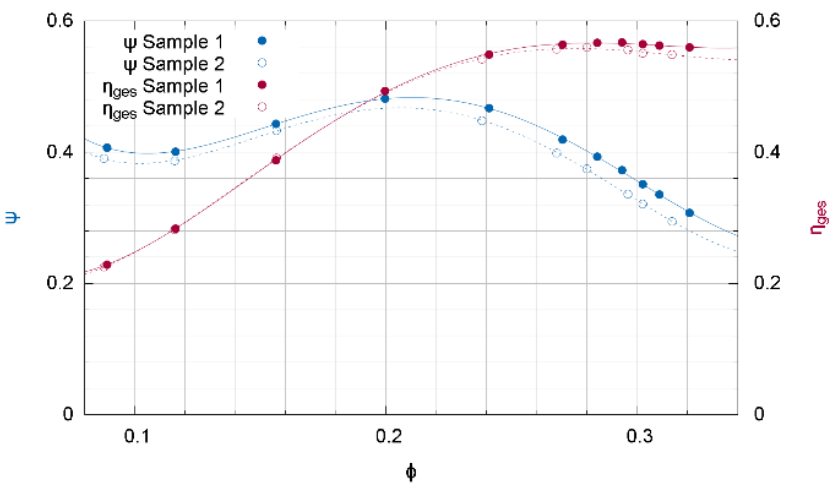

Fig. 8. Flow number vs. pressure number and total efficiency of Sample 1 (continuous line and filled points) and Sample 2 (dashed line and empty points).

The maximum efficiency $\eta_{g e s}=\frac{Q \Delta p_{t}}{P_{e l}}$ is obtained at $\varphi=0.28$. The resulting pressure number is $\Psi=0.4$. In comparison to the parameters described in section "Test Samples" design point and best point of operation are fitting sufficiently. The measurement results for Sample 3 and Sample 4 are similar to the results of Sample 2.

The subsequent measurements have been carried out using a constant volume flow at $0,486 \mathrm{~m}^{3} / \mathrm{s}$.

\subsection{Noise}

The acoustic measurements are completely carried out with rotational beamforming. Orthogonal as well as functional beamforming was applied in separate steps for increasing the dynamic range.

Acoustic images of the $5 \mathrm{kHz}$ and the $2 \mathrm{kHz}$ third octave band are shown below. In accordance to section "Functional Beamforming" the measured signals have to be rotated virtually with the rotational speed according to the rotational direction of the specific impeller. Due to the contra rotating working principle of impeller $\mathrm{A}$ and impeller B two acoustic images have to be calculated at every third octave band and for each test sample.

Fig. 9 depicts the acoustic images of the $5 \mathrm{kHz}$ third octave band. The upper row shows the impeller A (direction of rotation: clockwise), and the lower row shows the impeller B (direction of rotation: counterclockwise). Each of the four columns represents one sample, starting at the left column with Sample 1.

In Fig. 9 the rotating sound sources at impeller $A$ and impeller B are clearly visible. Corresponding to the amount of blades at impeller A, seven single aero-acoustic sources can be localised. At impeller B five single aeroacoustic sources can be localised.

In case of Sample 1 and Sample 3 the aero-acoustic sound sources of impeller A (small vanes) are always located at the trailing edges and in the middle of the span. At Sample 2 and Sample 4 the aero-acoustic sound sources are located in the middle between the leading and the trailing edges. The source location is close to the hub in radial direction. The colours are correlated with the sound pressure level.

As one may see, the noise level is attenuated by applying trailing edge serrations (Samples 2 and 4). 
Therefore, it was assumed that the noise of Sample 3 should be the same as for Sample 1 because of the same blades. In this special case the same level was not achieved. The underlying effects have to be investigated in further experiments.

Of impeller B (wide vanes) the dominant aeroacoustic sound source is the trailing edge for Samples 1 and 2. Contrary to Samples 1 and 2 (straight leading edge), Samples 3 and 4 (sinusoidal leading edge) have the highest amount of noise emission at the leading edge. Obviously, a sinusoidal leading edge leads to a change of the most important noise source. At this point it cannot be distinguished whether the noise at the trailing edge is diminished in comparison to the leading edge or the noise source is shifted from the trailing to the leading edge. Furthermore, the implementation of serrations at the trailing edges at the first impeller are leading to a noise reduction of the subsequent impeller, as being visible by comparison of Samples 2 and 4. The colours are indicating this noise reduction in both cases. The measured differences in the sound pressure levels between Samples 1 and 2 as well as between Samples 3 and 4 is in the range of $1 \mathrm{~dB}$ for impeller $\mathrm{B}$.

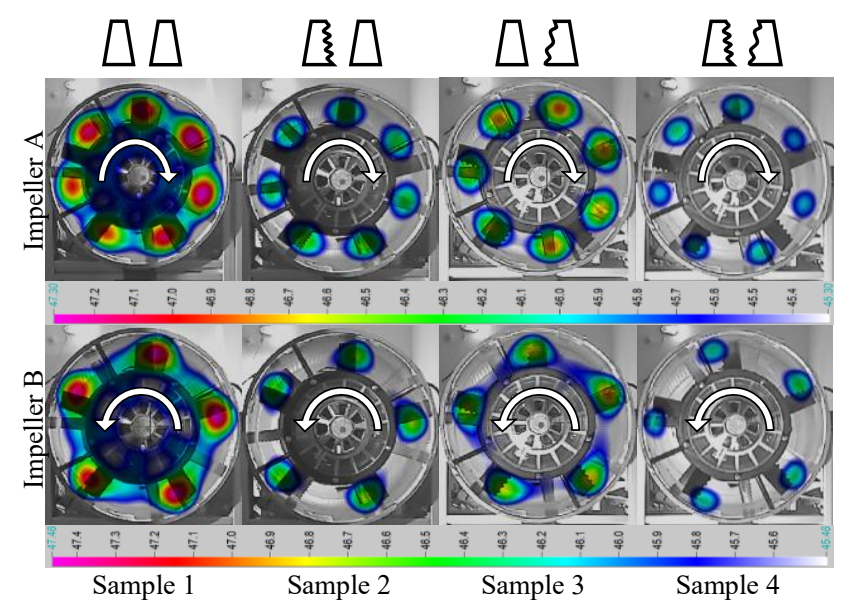

Fig. 9. Acoustic image with color-coded sound pressure level of the $2 \mathrm{kHz}$ third octave band for the different samples (horizontally oriented) and impeller A (top row) and impeller B (bottom row)

Impeller B exhibits nearly the same acoustic behaviour for both third octave bands of $2 \mathrm{kHz}$ and $5 \mathrm{kHz}$ (compare bottom rows of Fig. 9 and Fig. 10). Five noise sources can be identified clearly.

Contrary to the observations at $5 \mathrm{kHz}$ third octave band no specific noise sources could be detected on impeller A. The acoustic image shows a circular sound source. The noise emission of the impeller A is probably significantly lower than those emission of impeller B. Hence, the noise emission of impeller B masks the noise sources from impeller A. These sources from impeller B appear "smeared" in the acoustic pictures since the rotational filter applied with the speed and direction of impeller A, while they actually belong to impeller B.

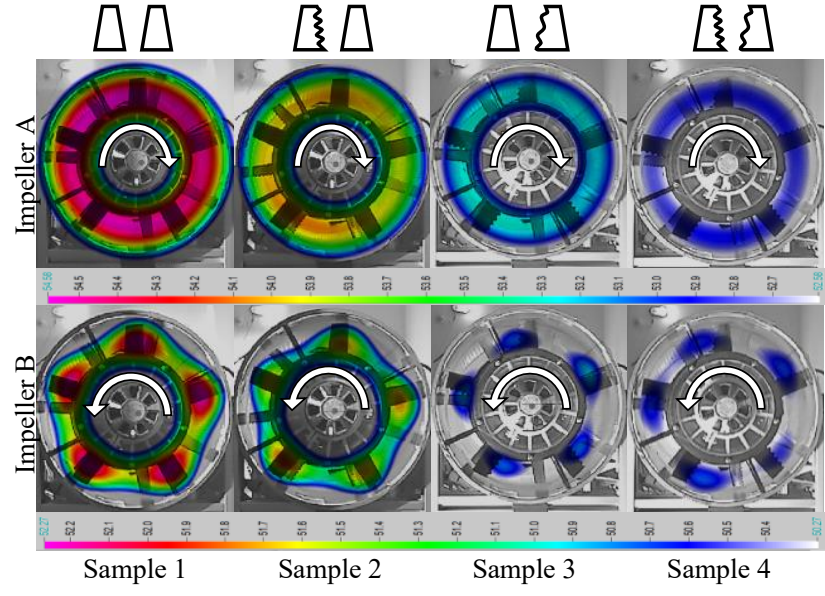

Fig. 10. Acoustic image with color-coded sound pressure level of the $2 \mathrm{kHz}$ third octave band for the different samples (horizontally oriented) and impeller A (top row) and impeller B (bottom row)

The differences in the sound pressure levels for the third octave frequencies of Samples 2, 3 and 4 to Sample 1 are presented in Fig. 11. Although the differences are low, there is a tendency of reduced noise emission for frequencies higher than $800 \mathrm{~Hz}$. The highest noise reduction is performed by Sample 4 at high frequencies. Instead low frequency noise reduction is performed by Sample 2 .

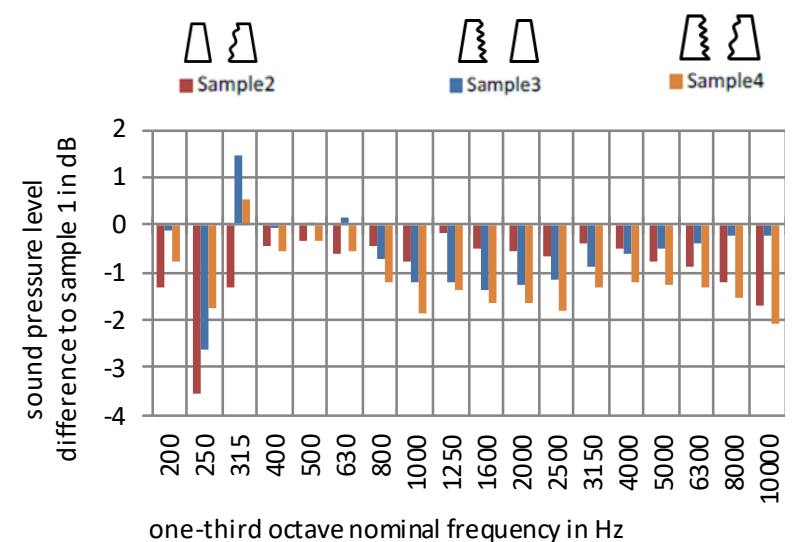

Fig. 11. Differences in the sound pressure level between Sample 1 and Sample 2 to Sample 4

\subsection{Measurement Uncertainty}

The uncertainty of the performed measurements depend among other influences on the used beamforming algorithm. In [18] Sarradj evaluates the influence of different formulations of the steering vectors on the source strength and position for a three-dimensional scenario. This of course concerns the absolute source strength.

In our case the relative certainty of the measurements are of higher interest. The rotational speed of the fans are rather stable. Therefore the aero-acoustic sound induced by the impellers is relatively stable. Furthermore the measurement conditions (test stand, camera position, working point etc.) could be reproduced. Consequently 
one can assume that the evaluated process is highly constant, though stochastic. To avoid further deviations the integration interval for the source evaluation has been chosen quite long ( 32 seconds). This leads to a reliable averaging of the sound field within this time period and therefore a low measurement uncertainty when comparing different types if impellers.

To get an impression of the uncertainty level the complete measurement signal of 32 seconds length has been evaluated in 2 seconds intervals. For each interval the source strength was calculated using rotational beamforming. This was evaluated exemplarily for the acoustic image in the upper left corner of Fig. 9 (both impellers with standard vanes, rotating clockwise, $5 \mathrm{kHz}$ third octave band). Table 1 shows the results for 16 intervals of 2 seconds length:

Table 1. Source strength of the same acoustic sound source in various two-seconds intervals

\begin{tabular}{|l|l|l|l|}
\hline Interval & $\begin{array}{l}\text { Source } \\
\text { Strength }\end{array}$ & Interval & $\begin{array}{l}\text { Source } \\
\text { Strength }\end{array}$ \\
\hline $0-2 \mathrm{sec}$ & $47,52 \mathrm{~dB}$ & $16-18 \mathrm{sec}$ & $47,28 \mathrm{~dB}$ \\
\hline $2-4 \mathrm{sec}$ & $47,41 \mathrm{~dB}$ & $18-20 \mathrm{sec}$ & $47,29 \mathrm{~dB}$ \\
\hline $4-6 \mathrm{sec}$ & $47,39 \mathrm{~dB}$ & $20-22 \mathrm{sec}$ & $47,47 \mathrm{~dB}$ \\
\hline $6-8 \mathrm{sec}$ & $47,30 \mathrm{~dB}$ & $22-24 \mathrm{sec}$ & $47,44 \mathrm{~dB}$ \\
\hline $8-10 \mathrm{sec}$ & $47,19 \mathrm{~dB}$ & $24-26 \mathrm{sec}$ & $47,40 \mathrm{~dB}$ \\
\hline $10-12 \mathrm{sec}$ & $47,22 \mathrm{~dB}$ & $26-28 \mathrm{sec}$ & $47,36 \mathrm{~dB}$ \\
\hline $12-14 \mathrm{sec}$ & $47,28 \mathrm{~dB}$ & $28-30 \mathrm{sec}$ & $47,41 \mathrm{~dB}$ \\
\hline $14-16 \mathrm{sec}$ & $47,34 \mathrm{~dB}$ & $30-32 \mathrm{sec}$ & $47,26 \mathrm{~dB}$ \\
\hline
\end{tabular}

The source strength within the complete 32 seconds interval has been calculated with $47,30 \mathrm{~dB}$. When averaging the 16 two-seconds intervals we get an average of $47,34 \mathrm{~dB}$ with a standard deviation of $0,09 \mathrm{~dB}$. This difference might result from the fact that with the 32 second interval also effects of the 2 second interval limits are averaged.

Of course the uncertainty level will differ from measurement to measurement and when taking different interval lengths into account. This could be part of future investigations. The uncertainty level in the residual measurements can be assumed as similar since all boundary conditions can be reproduced with high precision.

\section{Conclusion}

Different suggestions of literature on how to reduce the noise emission of fans were investigated. Therefore, a contra-rotating fan with different modifications of the blades was used. A permutation of standard blades, serrations at the trailing edge of the first impeller and sinusoidal modifications at the leading edge of the second impeller were designed and investigated.

The noise measurements were carried out with an Acoustic Camera. Because of the rotation of the impellers a rotational beamforming algorithm was developed for the measurements.

It could be shown that it is possible to locate sound sources on rotating blades of a contra rotating axial fan.
Despite the contra rotating impellers, sound sources can be assigned to the specific impeller. It is also possible to localise the specific location of the sound source on the blades. However, if the noise emission of one impeller is significantly less than the contra rotating impeller, the sound sources will be hidden behind a circular sound source.

Generally, it can be stated that the sound emission will be attenuated by using serrations at the trailing edge. In the third octave bands of $2 \mathrm{kHz}$ and $5 \mathrm{kHz}$ the reduction is about $1 \mathrm{~dB}$, each.

Using leading edges, the measurement results indicate noise source alterations. Until now it can not be clearly established how the interaction between the two contrarotating impellers effects the location and the emission of the rotating sound sources.

For a better understanding of the effects further investigations will be done. The next step will be the measurement of the flow structure around the couple of two blades. The correlation between the flow structure around the blade couples and the noise emission are of specific interest. For this purpose a non-invasive measurement technic, the Particle-Image-Velocimetry will be used.

It still requires further investigations for both the development of measurement methods to locate sound sources in contra rotating systems and to increase the understanding of the mechanism of the sound emission on the rotating blades of fans.

\section{Acknowledgements}

The research project is funded by the German Federal Ministry of Economic Affairs and Energy (BMWi) under the title "Primary noise reduction on a contra-rotating axial fan" (MF150166).

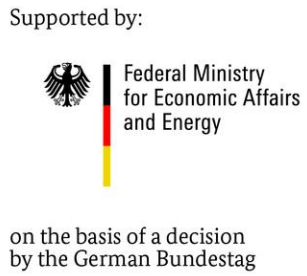

\section{Bibliography}

1. M. Kerscher, G. Heilmann, C. Puhle, R. Krause, C. Friebe, - Sound Source Localization on a Fast Rotating Fan Using Rotational Beamforming INTER-NOISE, the 46th International Congress and Exposition on Noise Control Engineering, 626 (1-8), Hong Kong, China, (2017)

2. A. Corsini, G. Delibra, Leading Edge Bumps in Ventilation Fans Paper No. GT2013-94853, pp. V004T10A007, doi:10.1115/GT2013-94853, (2013)

3. K. Hansen, et al, Reduction of Flow Induced Airfoil Tonal Noise using leading edge sinusoidal modifications Journal of Aircraft 11:4, 197-202, doi: 10.2514/3.59219, (1974) 
4. Alan S. Hersh, et al, Investigation of Acoustic Effects of Leading-Edge Serrations on Airfoils Acoustics Australia 172 - Vol. 40, No. 3, (2012

5. C. Polacsek, et al, Turbulence-airfoil interaction noise reduction using wavy leading edge: An experimental and numerical study Internoise, Osaka, Japan, (2011)

6. P.T Soderman, Aerodynamic effect of leading edge serrations a two-dimensional airfoil NASA TM X2643, (1972)

7. H. Johari, C. W. Henoch, D. Custodio, A. Levshin, Effects of Leading-Edge Protuberances on Airfoil Performance, AIAA Journal, Jg. 45, Nr. 11, S. 26342642 (2007)

8. Th. Carolus, Ventilatoren - Aerodynamischer Entwurf, Schallvorhersage, Konstruktion. 3. Auflage Springer Vieweg, ISBN 978-3-8348-2471-4, (2013)

9. F.M. Catalano, Airfoil self Noise Reduction by Application of different Types of Trailing Edge Serrations 28th International Congress of the Aeronautical Sciences, (2012)

10. M. S. Howe, Noise produced by a sawtooth trailing edge, Journal of The Acoustical Society of America, (1991)

11. E. Sarradj - Three-Dimensional Acoustic Source Mapping with Different Beamforming Steering Vector Formulations Advances in Acoustics and Vibration, (2012)

12. R.P. Dougherty - Functional beamforming Berlin Beamforming Conference 2014, (2014)

13. E. Sarradj - A fast signal subspace approach for the determination of absolute levels from phased microphone array measurements Journal of Sound and Vibration 329,1553-1569, (2010)

14. P. Sijtsma - CLEAN based on spatial source coherence International Journal of Aeroacoustics 6, 357-374, (2007)

15. CFturbo GmbH, - CFturbo Version 10.3, (2017)

16. R. Krause, C. Friebe, M. Kerscher, C, Puhle, Investigations on noise sources on a contra-rotating axial fan with different modifications, fan2018, Darmstadt (2018)

17. DIN EN ISO 5801 - Industrial fans - Performance testing using standardized airways (ISO 5801:2007, including Cor 1:2008); German version EN ISO 5801, (2008)

18. E. Sarradj, Three-Dimensional Acoustic Source Mapping with Different Beamforming Steering Vector Formulations; Advances in Acoustics and Vibration, Volume 2012 (2012), Article ID 292695, 12 pages, (2012) 